\title{
On a $q$-analog of some numbers and polynomials
}

Serkan Araci ${ }^{1 *}$, Erkan Ağyüz ${ }^{2}$ and Mehmet Acikgoz ${ }^{2}$

${ }^{\text {"Correspondence: }}$

mtsrkn@hotmail.com

${ }^{1}$ Department of Economics, Faculty

of Economics, Administrative and

Social Science, Hasan Kalyoncu

University, Gaziantep, 27410, Turkey

Full list of author information is

available at the end of the article

\begin{abstract}
In this paper, we introduce new $q$-analogs of the Changhee numbers and polynomials of the first kind and of the second kind. We also derive some new interesting identities related to the Stirling numbers of the first kind and of the second kind, the Euler polynomials of higher order and the $q$-analogs of Euler polynomials by applying the $p$-adic integrals method and some summation transform techniques. It turns out that some well-known results are derived as special cases.

MSC: $05 \mathrm{~A} 19 ; 11 \mathrm{~B} 68 ; 11 \mathrm{~B} 83$

Keywords: fermionic $p$-adic $q$-integral on $\mathbb{Z}_{p}$; Changhee polynomials; Pochhammer symbol; Stirling numbers of the first kind; Stirling numbers of the second kind; higher order Euler polynomials
\end{abstract}

\section{Introduction}

In mathematics, special functions (or special polynomials) are known as 'useful functions'. Because of their remarkable properties, special functions have been used for centuries. For instance, since they have numerous applications in astronomy, trigonometric functions have been studied for over a thousand years. Since then, the theory of special functions has been continuously developed with contributions by a host of mathematicians, including Euler, Legendre, Laplace, Gauss, Kummer, Eisenstein, Riemann, Ramanujan, and so on.

In the past years, the development of new special functions and of applications of special functions to new areas of mathematics have initiated a resurgence of interest in the $p$-adic analysis, $q$-analysis, analytic number theory, combinatorics, and so on. Moreover, in recent years, the various generalizations of the familiar special polynomials have been defined by using $p$-adic $q$-integral on $\mathbb{Z}_{p}$ and $p$-adic fermionic $q$-deformed integrals on $\mathbb{Z}_{p}$ introduced and investigated by Kim [1-4]. Srivastava and Todorov [5] derived an interesting extension of a representation for the generalized Bernoulli numbers in order to obtain interesting special cases considered earlier by Gould [6]. For more on these issues, e.g., see [6-21].

Let $p$ be chosen as a fixed odd prime number. Throughout this paper, we make use of the following notations. $\mathbb{Z}_{p}$ denotes the ring of integers, $\mathbb{Q}_{p}$ denotes the field of $p$-adic numbers, and $\mathbb{C}_{p}$ denotes the completion of the algebraic closure of $\mathbb{Q}_{p}$. The $p$-adic norm $|\cdot|_{p}$ is normalized by

$$
|p|_{p}=p^{-1}
$$

(c) 2015 Araci et al.; licensee Springer. This is an Open Access article distributed under the terms of the Creative Commons Attribution License (http://creativecommons.org/licenses/by/4.0), which permits unrestricted use, distribution, and reproduction in any medium, provided the original work is properly credited. 
Let $C\left(\mathbb{Z}_{p}\right)$ be the space of continuous functions on $\mathbb{Z}_{p}$. For $f \in C\left(\mathbb{Z}_{p}\right)$, the fermionic $p$ adic $q$-invariant integral on $\mathbb{Z}_{p}$ is defined by Kim $[1,2]$, as follows:

$$
I_{-q}(f)=\int_{\mathbb{Z}_{p}} f(x) d \mu_{-q}(x)=\lim _{n \rightarrow \infty} \sum_{x=0}^{p^{n}-1} f(x) \frac{(-q)^{x}}{\left[p^{n}\right]_{-q}} .
$$

It follows from (1.1) that

$$
q I_{-q}\left(f_{1}\right)+I_{-q}(f)=[2]_{q} f(0),
$$

where $f_{1}(x):=f(x+1)$. Obviously

$$
\lim _{q \rightarrow 1} I_{-q}(f)=I_{-1}(f)=\lim _{n \rightarrow \infty} \sum_{x=0}^{p^{n}-1} f(x)(-1)^{x} \quad c f .[22,23] .
$$

In [23], the Changhee polynomials are defined by substituting $f(x)=(1+t)^{x}$ into (1.3) with the case $|t|_{p}<p^{-\frac{1}{p-1}}$, as follows:

$$
\begin{aligned}
\int_{\mathbb{Z}_{p}}(1+t)^{x+y} d \mu_{-1}(y) & =\sum_{n=0}^{\infty}\left(\int_{\mathbb{Z}_{p}}(x+y)_{n} d \mu_{-1}(y)\right) \frac{t^{n}}{n !} \\
& =\sum_{n=0}^{\infty} C h_{n}(x) \frac{t^{n}}{n !} \\
& =\frac{2}{2+t}(1+t)^{x},
\end{aligned}
$$

where $(x)_{n}$ is known as the Pochhammer symbol (or decreasing factorial) defined by

$$
\begin{aligned}
(x)_{n} & =x(x-1) \cdots(x-n+1) \\
& =\sum_{k=0}^{n} S_{1}(n, k) x^{k}
\end{aligned}
$$

and here $S_{1}(n, k)$ is the Stirling number of the first kind (see [23-25]).

In [23], Kim et al. introduced using $p$-adic integral techniques the idea that the Changee numbers are closely related to the Euler numbers as follows:

$$
E_{m}=\sum_{n=0}^{m} C h_{n} S_{2}(n, m)
$$

where $S_{2}(n, m)$ is the Stirling number of the second kind defined by the following generating series:

$$
\sum_{n=m}^{\infty} S_{2}(n, m) \frac{t^{n}}{n !}=\frac{\left(e^{t}-1\right)^{m}}{m !} \quad c f .[23,24]
$$


In [15], Srivastava extended the Stirling numbers of the second kind to $\lambda$-Stirling numbers of the second kind as follows:

$$
\frac{\left(\lambda e^{t}-1\right)^{m}}{m !}=\sum_{n=0}^{\infty} \mathcal{S}(n, m ; \lambda) \frac{t^{n}}{n !} \quad\left(m \in \mathbb{N}^{*} \text { and } \lambda \in \mathbb{C}\right)
$$

with, of course,

$$
\mathcal{S}(n, m ; 1):=S_{2}(n, m)
$$

In [2], the Euler polynomials of (real or complex) order $\alpha$

$$
E_{n}^{(\alpha)}(x)
$$

(sometimes called the Euler polynomials of higher order) are introduced by the following generating function:

$$
\left(\frac{2}{e^{t}+1}\right)^{\alpha} e^{x t}=\sum_{n=0}^{\infty} E_{n}^{(\alpha)}(x) \frac{t^{n}}{n !} \quad(|t|<\pi)
$$

with, of course,

$$
E_{n}^{(1)}(x):=E_{n}(x) \quad \text { and } \quad E_{n}^{(\alpha)}(0):=E_{n}^{(\alpha)}
$$

where $E_{n}(x)$ and $E_{n}^{(\alpha)}$ are the $n$th Euler polynomials and the $n$th Euler numbers of order $\alpha$.

Recently, Kim et al. have studied the various generalizations of Changhee polynomials cf. [24, 26, 27]. Our $q$-analogs of the Changhee numbers and polynomials in the present paper are different from Kim et al.'s $q$-analogs of the Changhee numbers and polynomials. In this paper, we introduce a $q$-analog of the Changhee polynomials and derive some new interesting identities.

\section{On a $q$-analog of Changhee numbers and polynomials}

Let us now consider the following $p$-adic $q$-integral representation in accordance with the Pochhammer symbol:

$$
\int_{\mathbb{Z}_{p}} q^{-y}(x+y)_{n} d \mu_{-q}(y) \quad\left(n \in \mathbb{Z}_{+}=\mathbb{N} \cup\{0\}\right) .
$$

From (2.1), we have

$$
\begin{aligned}
\sum_{n=0}^{\infty}\left(\int_{\mathbb{Z}_{p}} q^{-y}(x+y)_{n} d \mu_{-q}(y)\right) \frac{t^{n}}{n !} & =\int_{\mathbb{Z}_{p}} q^{-y}\left(\sum_{n=0}^{\infty}\left(\begin{array}{c}
x+y \\
n
\end{array}\right) t^{n}\right) d \mu_{-q}(y) \\
& =\int_{\mathbb{Z}_{p}} q^{-y}(1+t)^{x+y} d \mu_{-q}(y),
\end{aligned}
$$

where $t \in \mathbb{C}_{p}$ with $|t|_{p}<p^{-\frac{1}{p-1}}$. Applying (1.2) to (2.2) gives

$$
\int_{\mathbb{Z}_{p}} q^{-y}(1+t)^{x+y} d \mu_{-q}(y)=\frac{1+q}{1+(1+t)^{-1}}(1+t)^{x-1} .
$$


Let

$$
F_{q}(x, t)=\frac{1+q}{1+(1+t)^{-1}}(1+t)^{x-1} .
$$

Then

$$
\lim _{q \rightarrow 1} F_{q}(x, t)=\frac{2}{2+t}(1+t)^{x} .
$$

Notice that $F_{q}(x, t)$ seems to be a new $q$-extension of the generating function for aforementioned Changhee polynomials of the first kind. Therefore, from (1.4) and (2.3), we obtain the following definition.

Definition 1 Let $F_{q}(x, t)=\sum_{n=0}^{\infty} C h_{n}(x \mid q) \frac{t^{n}}{n !}$, where $C h_{n}(x \mid q)$ is called a $q$-analog of the $n$th Changhee polynomials of the first kind. Then we have for $n \geq 0$

$$
\sum_{n=0}^{\infty} C h_{n}(x \mid q) \frac{t^{n}}{n !}=\frac{1+q}{1+(1+t)^{-1}}(1+t)^{x-1}
$$

Moreover,

$$
C h_{n}(x \mid q)=\int_{\mathbb{Z}_{p}} q^{-y}(x+y)_{n} d \mu_{-q}(y) .
$$

In the case $x=0$ in Definition 1, we have $C h_{n}(0 \mid q):=C h_{n}(q)$, which stands for the $q$ analog of the $n$th Changhee numbers of the first kind. It follows from (1.4) and Definition 1 that

$$
\frac{1+q}{2} C h_{n}(x)=C h_{n}(x \mid q) .
$$

Equation (2.4) shows that our $q$-analog of the Changhee polynomials of the first kind is closely related to the Changhee polynomials. From (1.5) we have

$$
C h_{n}(q)=\sum_{k=0}^{n} S_{1}(n, k) E_{k}(q),
$$

where $E_{k}(q)$ are the $q$-Euler polynomials derived from

$$
E_{k}(q)=\int_{\mathbb{Z}_{p}} q^{-y} y^{k} d \mu_{-q}(y) .
$$

From (1.5), we have

$$
\begin{aligned}
C h_{n}(x \mid q) & =\int_{\mathbb{Z}_{p}} q^{-y}(x+y)_{n} d \mu_{-q}(y) \\
& =\sum_{k=0}^{n} S_{1}(n, k) E_{k}(x \mid q),
\end{aligned}
$$


where $E_{k}(x \mid q)$ are the $q$-Euler polynomials introduced by

$$
E_{k}(x \mid q)=\int_{\mathbb{Z}_{p}} q^{-y}(x+y)^{k} d \mu_{-q}(y) .
$$

From Definition 1, we have

$$
\begin{aligned}
\frac{1+q}{q e^{t}+1} & =\sum_{n=0}^{\infty} C h_{n}(q) \frac{1}{n !}\left(\frac{e^{t}}{q}-1\right)^{n} \\
& =\sum_{n=0}^{\infty} C h_{n}(q) \frac{1}{n !} n ! \sum_{m=n}^{\infty} S_{2}(m, n) \frac{(t-\log q)^{m}}{m !} \\
& =\sum_{m=0}^{\infty}\left(\sum_{n=0}^{m} C h_{n}(q) S_{2}(m, n)\right) \frac{(t-\log q)^{m}}{m !}
\end{aligned}
$$

It follows from (2.7) that

$$
\sum_{m=0}^{\infty}\left(\frac{1+q}{1+q^{2}} E_{m}\left(q^{2}\right)\right) \frac{t^{m}}{m !}=\sum_{m=0}^{\infty}\left(\sum_{n=0}^{m} C h_{n}(q) S_{2}(m, n)\right) \frac{t^{m}}{m !} .
$$

When we compare the coefficients $\frac{t^{n}}{n !}$ of both sides of the above we have

$$
\frac{1+q}{1+q^{2}} E_{m}\left(q^{2}\right)=\sum_{n=0}^{m} C h_{n}(q) S_{2}(m, n) .
$$

Therefore, we obtain the following theorem.

Theorem 1 For $m \geq 0$, we have

$$
\frac{1+q}{1+q^{2}} E_{m}\left(q^{2}\right)=\sum_{n=0}^{m} C h_{n}(q) S_{2}(m, n) .
$$

The increasing factorial sequence is known as

$$
x^{(n)}=x(x+1)(x+2) \cdots(x+n-1) \quad\left(n \in \mathbb{N}^{*}\right) .
$$

For more information as regards this sequence, see [11, 23, 24, 26, 27].

Let us define the $q$-analog of the Changhee numbers of the second kind as follows:

$$
\widehat{C h}_{n}(q)=\int_{\mathbb{Z}_{p}} q^{-y}(-y)_{n} d \mu_{-q}(y) \quad\left(n \in \mathbb{N}^{*}\right) .
$$

It is easy to observe that

$$
x^{(n)}=(-1)^{n}(-x)_{n}=\sum_{k=0}^{n} S_{1}(n, k)(-1)^{n-k} x^{k} .
$$


By virtue of (2.8) and (2.9), it leads to

$$
\begin{aligned}
\widehat{C h}_{n}(q) & =\int_{\mathbb{Z}_{p}} q^{-y}(-y)_{n} d \mu_{-q}(y) \\
& =\int_{\mathbb{Z}_{p}} q^{-y} y^{(n)}(-1)^{n} d \mu_{-q}(y) \\
& =\sum_{k=0}^{n} S_{1}(n, k)(-1)^{k} E_{k}(q) .
\end{aligned}
$$

Thus, we state the following theorem.

Theorem 2 The following holds true:

$$
\widehat{C h}_{n}(q)=\sum_{k=0}^{n} S_{1}(n, k)(-1)^{k} E_{k}(q) .
$$

Let us now consider the generating function of the $q$-Changhee numbers of the second kind as follows:

$$
\begin{aligned}
\sum_{n=0}^{\infty} \widehat{C h}_{n}(q) \frac{t^{n}}{n !} & =\sum_{n=0}^{\infty}\left(\int_{\mathbb{Z}_{p}} q^{-y}(-y)_{n} d \mu_{-q}(y)\right) \frac{t^{n}}{n !} \\
& =\int_{\mathbb{Z}_{p}} q^{-y}\left(\sum_{n=0}^{\infty}\left(\begin{array}{c}
-y \\
n
\end{array}\right) t^{n}\right) d \mu_{-q}(y) \\
& =\int_{\mathbb{Z}_{p}} q^{-y}(1+t)^{-y} d \mu_{-q}(y),
\end{aligned}
$$

in which $\int_{\mathbb{Z}_{p}} q^{-y}(1+t)^{-y} d \mu_{-q}(y)$ equals

$$
\frac{(1+q)}{1+(1+t)^{-1}} \text {. }
$$

Then, combining (2.11) with (2.12), we state the following definition.

Definition 2 For $n \geq 0$, we have

$$
\begin{aligned}
\sum_{n=0}^{\infty} \widehat{C h}_{n}(q) \frac{t^{n}}{n !} & =\int_{\mathbb{Z}_{p}} q^{-y}(1+t)^{-y} d \mu_{-q}(y) \\
& =\frac{(1+q)}{1+(1+t)^{-1}} .
\end{aligned}
$$

Let us consider the $q$-Changhee polynomials of the second kind as follows:

$$
\frac{1+q}{2+t}(1+t)^{1-x}=\sum_{n=0}^{\infty} \widehat{C h}_{n}(x \mid q) \frac{t^{n}}{n !}
$$

Combining (2.4) with (2.13) at the value $x=1$, we have

$$
\widehat{C h}_{n}(1 \mid q)=\frac{1+q}{2} C h_{n}=C h_{n}(q) \text {. }
$$


It follows from (2.13) that

$$
\int_{\mathbb{Z}_{p}} q^{-y}(1+t)^{-x-y} d \mu_{-q}(y)=\sum_{n=0}^{\infty} \widehat{C h}_{n}(x \mid q) \frac{t^{n}}{n !}
$$

From (2.14) gives

$$
\begin{aligned}
\widehat{C h}_{n}(x \mid q) & =\int_{\mathbb{Z}_{p}} q^{-y}(-x-y)_{n} d \mu_{-q}(y) \\
& =\sum_{k=0}^{n}(-1)^{k} S_{1}(n, k) E_{k}(x \mid q) \quad(n \geq 0) .
\end{aligned}
$$

Then, by (2.15), we have the following theorem.

Theorem 3 The following holds true:

$$
\widehat{C h}_{n}(x \mid q)=\sum_{k=0}^{n}(-1)^{k} S_{1}(n, k) E_{k}(x \mid q) \quad(n \geq 0) .
$$

From (2.13) and (2.14), we have

$$
\begin{aligned}
q^{1-x}\left(\frac{1+q}{q e^{t}+1}\right) e^{(1-x) t} & =\sum_{n=0}^{\infty} \widehat{C h}_{n}(x \mid q) \frac{1}{n !}\left(q e^{t}-1\right)^{n} \\
& =\sum_{n=0}^{\infty} \widehat{C h}_{n}(x \mid q) \sum_{m=n}^{\infty} \mathcal{S}(m, n ; q) \frac{t^{m}}{m !} \\
& =\sum_{m=0}^{\infty}\left(\sum_{n=0}^{m} \widehat{C h}_{n}(x \mid q) \mathcal{S}(m, n ; q)\right) \frac{t^{m}}{m !}
\end{aligned}
$$

Further

$$
\begin{aligned}
q^{1-x} \int_{\mathbb{Z}_{p}} e^{(1-x+y) t} d \mu_{-q}(y) & =\sum_{n=0}^{\infty} \widehat{C h}_{n}(x \mid q) \frac{\left(q e^{t}-1\right)^{n}}{n !} \\
& =\sum_{m=0}^{\infty}\left(\sum_{n=0}^{m} \widehat{C h}_{n}(x \mid q) \mathcal{S}(m, n ; q)\right) \frac{t^{m}}{m !}
\end{aligned}
$$

Therefore, by (2.16) and (2.17), we obtain the following theorem.

Theorem 4 The following equality holds true:

$$
q^{1-x} \mathcal{E}_{m}(1-x \mid q)=(-1)^{n} q^{1-x} \mathcal{E}_{m}\left(x \mid q^{-1}\right)=\sum_{n=0}^{m} \widehat{C h}_{n}(x \mid q) \mathcal{S}(m, n ; q)
$$

where $\mathcal{E}_{m}(x \mid q)$ may be called the mth q-Euler polynomials

$$
\mathcal{E}_{m}(x \mid q)=\int_{\mathbb{Z}_{p}}(x+y)^{m} d \mu_{-q}(y)
$$


because

$$
\lim _{q \rightarrow 1} \mathcal{E}_{m}(x \mid q):=E_{m}(x)
$$

From Definition 1 and (2.15) we have

$$
\begin{aligned}
(-1)^{n} \frac{C h_{n}(q)}{n !} & =(-1)^{n} \int_{\mathbb{Z}_{p}} q^{-y}\left(\begin{array}{l}
y \\
n
\end{array}\right) d \mu_{-q}(y) \\
& =\int_{\mathbb{Z}_{p}} q^{-y}\left(\begin{array}{c}
-y+n-1 \\
n
\end{array}\right) d \mu_{-q}(y) \\
& =\sum_{m=0}^{n}\left(\begin{array}{c}
n-1 \\
n-m
\end{array}\right) \int_{\mathbb{Z}_{p}} q^{-y}\left(\begin{array}{l}
-y \\
m
\end{array}\right) d \mu_{-q}(y) \\
& =\sum_{m=1}^{n}\left(\begin{array}{c}
n-1 \\
m-1
\end{array}\right) \frac{\widehat{C h}_{m}(q)}{m !}
\end{aligned}
$$

and

$$
\begin{aligned}
(-1)^{n} \frac{\widehat{C h}_{n}(q)}{n !} & =(-1)^{n} \int_{\mathbb{Z}_{p}} q^{-y}\left(\begin{array}{c}
-y \\
n
\end{array}\right) d \mu_{-q}(y) \\
& =\int_{\mathbb{Z}_{p}} q^{-y}\left(\begin{array}{c}
y+n-1 \\
n
\end{array}\right) d \mu_{-q}(y) \\
& =\sum_{m=0}^{n}\left(\begin{array}{c}
n-1 \\
n-m
\end{array}\right) \int_{\mathbb{Z}_{p}} q^{-y}\left(\begin{array}{l}
y \\
m
\end{array}\right) d \mu_{-q}(x) \\
& =\sum_{m=1}^{n}\left(\begin{array}{c}
n-1 \\
m-1
\end{array}\right) \frac{\widehat{C h}_{m}(q)}{m !} .
\end{aligned}
$$

Therefore, we get the following theorem.

Theorem 5 The following holds:

$$
(-1)^{n} \frac{C h_{n}(q)}{n !}=\sum_{m=1}^{n}\left(\begin{array}{c}
n-1 \\
m-1
\end{array}\right) \frac{\widehat{C h}_{m}(q)}{m !}
$$

and

$$
(-1)^{n} \frac{\widehat{C h}_{n}(q)}{n !}=\sum_{m=1}^{n}\left(\begin{array}{c}
n-1 \\
m-1
\end{array}\right) \frac{\widehat{C h}_{m}(q)}{m !}
$$




\section{Author details}

'Department of Economics, Faculty of Economics, Administrative and Social Science, Hasan Kalyoncu University, Gaziantep, 27410, Turkey. ${ }^{2}$ Department of Mathematics, Faculty of Arts and Science, University of Gaziantep, Gaziantep, 27310 , Turkey.

\section{Acknowledgements}

The authors are grateful to the comments of the referees, which have improved the quality of the paper substantially.

Received: 23 September 2014 Accepted: 22 December 2014 Published online: 20 January 2015

\section{References}

1. Kim, T: $q$-Euler numbers and polynomials associated with $p$-adic $q$-integrals. J. Nonlinear Math. Phys. 14(1), 15-27 (2007)

2. Kim, T: Some identities on the $q$-Euler polynomials of higher order and $q$-Stirling numbers by the fermionic $p$-adic integral on $\mathbb{Z}_{p}$. Russ. J. Math. Phys. 16, 484-491 (2009)

3. Kim, T, Dolgy, DV, Kim, DS, Rim, S-H: A note on the identities of special polynomials. Ars Comb. 113A, 97-106 (2014)

4. Kim, DS, Kim, T: Higher-order Cauchy of the second kind and poly-Cauchy of the second kind mixed type polynomials. Ars Comb. 115, 435-451 (2014)

5. Srivastava, HM, Todorov, PG: An explicit formula for the generalized Bernoulli polynomials. J. Math. Anal. Appl. 130, 509-513 (1988)

6. Gould, HW: Explicit formulas for Bernoulli numbers. Am. Math. Mon. 79, 44-51 (1972)

7. Araci, S, Acikgoz, M, Bagdasaryan, A, Şen, E: The Legendre polynomials associated with Bernoulli, Euler, Hermite and Bernstein polynomials. Turk. J. Anal. Number Theory 1(1), 1-3 (2013)

8. Araci, S, Acikgoz, M, Sen, E: On the von Staudt-Clausen's theorem associated with q-Genocchi numbers. Appl. Math. Comput. 247, 780-785 (2014)

9. Andrews, GE, Askey, R, Roy, R: Special Functions. Encyclopedia of Mathematics and Its Applications, vol. 71. Cambridge University Press, Cambridge (1999)

10. Kim, T: q-Volkenborn integration. Russ. J. Math. Phys. 9(3), 288-299 (2002)

11. Kim, T: An invariant $p$-adic integral associated with Daehee numbers. Integral Transforms Spec. Funct. 13(1), 65-69 (2002)

12. Ryoo, CS, Kim, T, Agarwal, RP: Exploring the multiple Changhee q-Bernoulli polynomials. Int. J. Comput. Math. 82(4), 483-493 (2005)

13. Simsek, Y: Special functions related to Dedekind-type DC-sums and their applications. Russ. J. Math. Phys. 17(4), 495-508 (2010)

14. Srivastava, HM: Some formulas for the Bernoulli and Euler polynomials at rational arguments. Math. Proc. Camb. Philos. Soc. 129, 77-84 (2000)

15. Srivastava, HM: Some generalizations and basic (or q-) extensions of the Bernoulli, Euler and Genocchi polynomials. Appl. Math. Inf. Sci. 5, 390-444 (2011)

16. Srivastava, HM, Choi, J: Zeta and q-Zeta Functions and Associated Series and Integrals. Elsevier, Amsterdam (2012)

17. He, Y, Wang, SJ: New formulae of products of the Frobenius-Euler polynomials. J. Inequal. Appl. 2014,261 (2014)

18. Araci, S, Acikgoz, M, Şen, E: On the extended Kim's $p$-adic $q$-deformed fermionic integrals in the $p$-adic integer ring. J. Number Theory 133, 3348-3361 (2013)

19. Araci, S, Bagdasaryan, A, Özel, C, Srivastava, HM: New symmetric identities involving q-zeta type functions. Appl. Math. Inf. Sci. 8, 2803-2808 (2014)

20. Carlitz, L: A note on Bernoulli and Euler polynomials of the second kind. Scr. Math. 25, 323-330 (1961)

21. Comtet, L: Advanced Combinatorics. Reidel, Dordrecht (1974)

22. Araci, S: Novel identities involving Genocchi numbers and polynomials arising from applications of umbral calculus. Appl. Math. Comput. 233, 599-607 (2014)

23. Kim, DS, Kim, T, Seo, JJ: A note on Changhee polynomials and numbers. Adv. Stud. Theor. Phys. 7(20), 993-1003 (2013)

24. Kim, T, Mansour, T, Rim, S-H, Seo, J-J: A note on q-Changhee polynomials and numbers. Adv. Stud. Theor. Phys. 8(1), 35-41 (2014)

25. Guo, B-N, Qi, F: Some identities and an explicit formula for Bernoulli and Stirling numbers. J. Comput. Appl. Math. 255, 568-579 (2014)

26. Kim, DS, Kim, T, Seo, J-J, Lee, S-H: Higher-order Changhee numbers and polynomials. Adv. Stud. Theor. Phys. 8(8), 365-373 (2014)

27. Dolgy, DV, Kim, T, Rim, S-H, Seo, J-J: A note on Changhee polynomials and numbers with q-parameter. Int. J. Math. Anal. 8(26), 1255-1264 (2014) 